\title{
Hund excitations and the efficiency of Mott solar cells
}

\author{
Francesco Petocchi, ${ }^{1}$ Sophie Beck, ${ }^{2}$ Claude Ederer, ${ }^{2}$ and Philipp Werner ${ }^{1}$ \\ ${ }^{1}$ Department of Physics, University of Fribourg, 1700 Fribourg, Switzerland \\ ${ }^{2}$ Materials Theory, ETH Zurich, Wolfgang-Pauli-Strasse 27, 8093 Zürich, Switzerland
}

(Received 25 January 2019; revised manuscript received 22 July 2019; published 26 August 2019)

\begin{abstract}
We study the dynamics of photoinduced charge carriers in semirealistic models of $\mathrm{LaVO}_{3}$ and $\mathrm{YTiO}_{3}$ polar heterostructures. It is shown that two types of impact ionization processes contribute to the carrier multiplication in these strongly correlated multiorbital systems: The first mechanism involves local spin state transitions, while the second mechanism involves the scattering of high-kinetic-energy carriers. Both processes act on the 10-fs timescale and play an important role in the harvesting of high-energy photons in solar cell applications. As a consequence, the optimal gap size for Mott solar cells is substantially smaller than for semiconductor devices.
\end{abstract}

DOI: 10.1103/PhysRevB.100.075147

\section{INTRODUCTION}

The development of efficient photovoltaic technologies is essential for sustainable energy production. Over the last decade, the emergence of perovskite solar cells [1,2] has caught the attention of many researchers. The efficiency of metal halide perovskite devices has risen rapidly, but practical issues like stability and toxicity still need to be solved [3]. Much less in the spotlight, but conceptually interesting, is the proposal to build solar cells which exploit the peculiar properties of correlated electron systems [4,5]. Reference [4] pointed out that charge carriers with high kinetic energy in smallgap Mott insulators can excite additional electrons across the gap $\Delta_{g}$ via impact ionization. Related mechanisms have also been discussed for light-sensitive organic compounds [6] and semiconducting quantum dots [7]. This provides a strategy for harvesting high-energy photons, which may potentially lift the efficiency of Mott insulating solar cells above the Shockley-Queisser (SQ) limit [8] for semiconductor solar cells. A promising system, $\mathrm{LaVO}_{3}$ (LVO) on top of $\mathrm{SrTiO}_{3}$ (STO), was identified by Assmann et al. [9]. It has a direct band gap of about $1.1 \mathrm{eV}$ and an internal electric field due to the polar nature of the LVO layers. Experimentally, however, the devices fabricated so far have shown a low efficiency due to a low mobility of the photocarriers [10,11]. Also, theoretically, it has been argued that the strong internal fields of these structures may result in carrier localization [12]. To properly assess the device characteristics of clean Mott insulating heterostructures, it is essential to analyze the impact ionization processes in realistic multiorbital systems and the charge separation process in the presence of an external voltage bias. Here, we compute the nonequilibrium processes in Mott solar cells using the nonequilibrium extension of dynamical mean-field theory (DMFT) [13] with a modified self-consistency which allows us to incorporate the essential aspects of the noninteracting band structure derived from density-functional theory (DFT). We perform an extensive analysis of the effect of a solarlike excitation on two compounds in their bulk phase, namely, LVO and $\mathrm{YTiO}_{3}$ (YTO) in their $d^{2}$ and $d^{1}$ configurations, respectively. YTO shares many of the correlation-induced properties with LVO but exhibits a smaller spectral gap [14,15]. The comparison of these two systems allows us to reveal the characteristic features of impact ionization and to assess its effect on the efficiency of Mott solar cells. We also study how the photoinduced charge carriers are separated in four-layer structures with an internal field gradient.

The paper is organized as follows. In Sec. II we describe the setup of the DFT calculations, the model, and the technique used to solve the model and the formulation of the light-matter interaction in multiorbital systems. In Sec. III we present our results for the impact ionization and carrier dynamics, while Sec. IV contains conclusions and perspectives.

\section{METHOD}

\section{A. DFT setup}

The DFT calculations are performed using the QUANTUM ESPRESSO package [16] with the Perdew, Burke, and Ernzerhof parametrization of the generalized gradient approximation (GGA) [17] to the exchange-correlation functional. The bulk material with space group symmetry Pbnm contains four symmetry-equivalent transition-metal sites within the primitive unit cell. For the heterostructures we fix the in-plane lattice parameters to the theoretical STO lattice constant and stack the films with the long orthorhombic axis parallel to the [001] growth direction, similar to Ref. [9]. Thereby, a glide plane $b$ parallel to the $c$ axis preserves the in-plane symmetry of the two transition-metal sites, such that we need to treat only one effective impurity problem per layer in the subsequent DMFT calculations. The $c$ component of the cell and all internal coordinates are fully relaxed until the force components are smaller than $1 \mathrm{mRy} / a_{0}\left(a_{0}\right.$ is the Bohr radius $)$ in the heterostructures $\left(0.1 \mathrm{mRy} / a_{0}\right.$ in the bulk) and all the components of the stress tensor are smaller than 0.5 kbar (0.1 kbar). We employ scalar-relativistic ultrasoft pseudopotentials with the following semicore states included in the valence: $3 s, 3 p$ for $\mathrm{V}$ and $\mathrm{Ti}, 4 s, 4 p$ for $\mathrm{Sr}$, and $5 s, 5 p$ for La. A plane-wave energy cutoff of $70 \mathrm{Ry}$ for the wave 
functions and 840 Ry for the charge density is used, along with a $6 \times 6 \times 4$ (bulk) or $8 \times 8 \times 4$ (heterostructure) Monkhorst-Pack $k$-point grid [18]. We use the Methfessel-Paxton smearing with a smearing parameter of $0.02 \mathrm{Ry}$ for the broadening of the electron occupations for atomic relaxations and 0.01 Ry for calculating the band structure. The construction of the low-energy tight-binding Hamiltonian is performed using the WANNIER90 code [19]. Three maximally localized Wannier functions (MLWFs) [20] for each transition-metal site are constructed from initial projections onto atomic $t_{2 g}$ orbitals. Since the energy window contains only the $t_{2 g}$-derived bands around the Fermi level, the resulting MLWFs exhibit $p$-like tails on the surrounding $\mathrm{O}$ atoms. In order to obtain a noninteracting effective tight-binding Hamiltonian $\mathcal{H}_{0}$ (without local splittings due to spin and orbital order) as input for the DMFT calculations the Wannier functions are constructed from a non-spin-polarized calculation. To estimate the potential gradient in the insulating (spin and orbitally ordered) layered system, we use spin-polarized GGA $+U$ calculations with $U_{\mathrm{V}}=3.0 \mathrm{eV}$ and $U_{\mathrm{Ti}}=9.8[9]$ and C-type antiferromagnetic order. In this case the in-plane lattice constants are set to the average of those of bulk LVO with GGA+ $U$.

\section{B. DMFT with simplified self-consistency}

The effective tight-binding Hamiltonian $\mathcal{H}_{0}$ of all our models consists of three predominantly $t_{2 g}$ bands, which are present near the Fermi level within an energy window comparable to the solar light. A comprehensive description of our systems should, in principle, include the $e_{g}$ orbitals, which lie above the Fermi level, as shown in the density of states (DOS) of the $e_{g}$-like MLWFs in Fig. 1. However, to reveal the role of impact ionization, in particular Hund excitations, it is sufficient to study the $t_{2 g}$ states. The local interaction is described by the three-orbital Slater-Kanamori Hamiltonian

$$
\begin{aligned}
\mathcal{H}_{U}= & U \sum_{\alpha} \hat{n}_{\alpha \uparrow} \hat{n}_{\alpha \downarrow}+U^{\prime} \sum_{\alpha \neq \beta} \hat{n}_{\alpha \uparrow} \hat{n}_{\beta \downarrow}+\left(U^{\prime}-J_{H}\right) \sum_{\sigma, \alpha<\beta} \hat{n}_{\alpha \sigma} \hat{n}_{\beta \sigma} \\
& +J_{H} \sum_{\alpha \neq \beta} c_{\alpha \uparrow}^{\dagger} c_{\beta \uparrow} c_{\beta \downarrow}^{\dagger} c_{\alpha \downarrow}+J_{H} \sum_{\alpha \neq \beta} c_{\alpha \uparrow}^{\dagger} c_{\beta \uparrow} c_{\alpha \downarrow}^{\dagger} c_{\beta \downarrow},
\end{aligned}
$$

where $c_{\alpha \sigma}\left(c_{\alpha \sigma}^{\dagger}\right)$ is the annihilation (creation) operator of an electron of spin $\sigma$ in orbital $\alpha$ and the interaction parameters are the local intraorbital (interorbital) Coulomb interaction $U\left(U^{\prime}=U-2 J_{H}\right)$ and Hund's coupling $J_{H}$. To address the time-dependent dynamics, we employ the nonequilibrium implementation of inhomogeneous DMFT [13,21], where every inequivalent site within the unit cell is mapped onto an impurity problem embedded in a self-consistently determined medium [22]. Within this approach, which assumes a local self-energy, the solution includes spatial correlations at the mean-field level but fully retains the dynamical energy and time-dependent fluctuations. The impurity problem of the time-dependent system is solved using a noncrossing approximation (NCA) solver $[23,24]$, which provides a qualitatively correct description of Mott insulating systems [25,26].

While an inhomogeneous nonequilibrium DMFT was previously used to study layered single-band systems [12,21], the full solution, with an explicit momentum summation, for two spins, three orbitals, and possibly more than one site
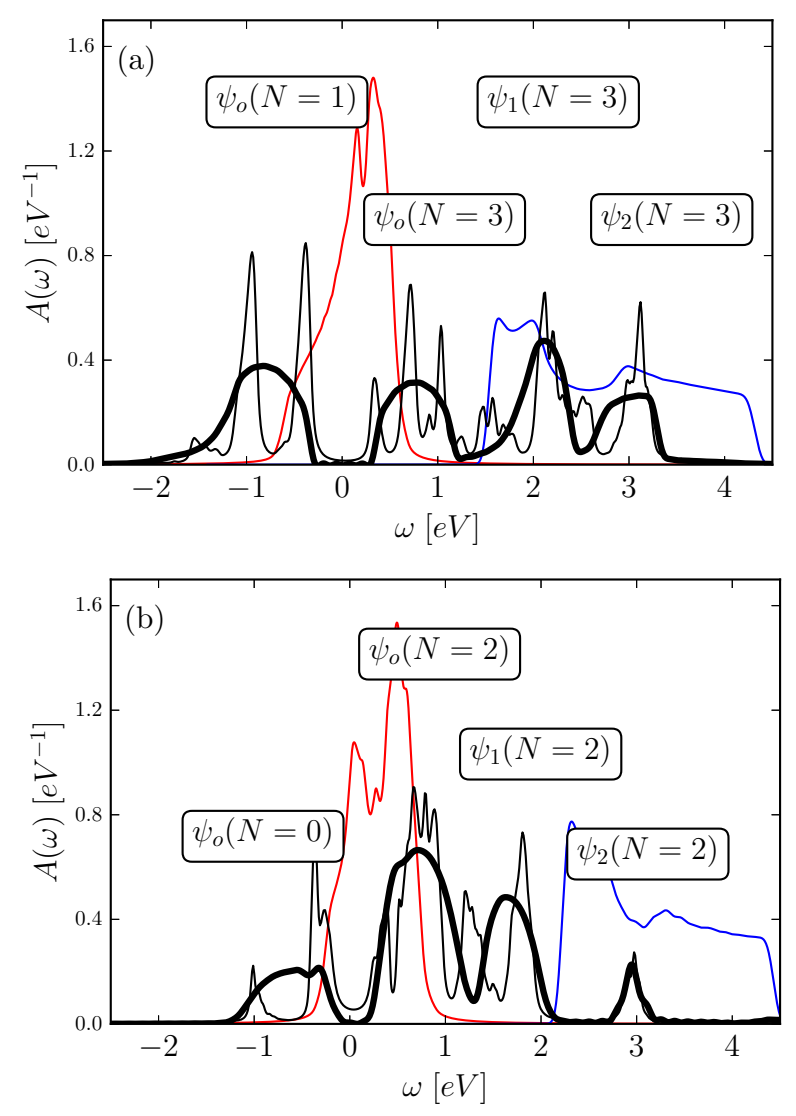

FIG. 1. A comparison between the total DMFT spectral functions at the transition-metal site computed with the full $\mathbf{k}$ summation and an ED solver (thin black lines) and those obtained with the approximate self-consistency and the NCA solver (thick black lines) for the $t_{2 g}$ orbitals in (a) LVO and (b) YTO. The labels show the indices of the available charge excitations associated with different eigenstates of the local $t_{2 g}$ Hamiltonian. The red and blue lines show, respectively, the DOS of the $t_{2 g}$ - and $e_{g}$-derived MLWFs based on the non-spin-polarized DFT calculation, illustrating the importance of electronic correlations in opening the gap. The two sets of MLWFs are obtained from two separate calculations within disjoint energy windows.

in the unit cell, would be plagued by severe memory limitations, restricting the simulations to short times. We hence implement a simplified self-consistency which is much more economical in terms of both computational cost and the memory requirement. The lattice information enters a DMFT calculation through the hybridization function $\hat{\Delta}$. Using the cavity method [22,27], $\hat{\Delta}$ for site $i$ can be expressed as

$$
\hat{\Delta}_{i}\left(t, t^{\prime}\right)=\sum_{j} \hat{h}_{i j}(t) \hat{G}_{j}^{[i]}\left(t, t^{\prime}\right) \hat{h}_{j i}^{*}\left(t^{\prime}\right),
$$

where $\hat{h}$ denotes the hopping amplitude and $\hat{G}_{j}^{[i]}$ is the Green's function at site $j$ in a lattice with site $i$ removed. All quantities are matrices in orbital and spin space. In a Mott insulating system, where the kinetic term is small, it is reasonable to replace the cavity $\hat{G}_{j}^{[i]}$ with the local Green's function $\hat{G}_{j}$, which results in a set of Bethe-like self-consistency equations [22] with $a b$ initio hopping parameters $h$, which we truncate at the second neighbor. To avoid the calculation of orbital 
off-diagonal components of $\hat{G}$, we rotate the hopping matrix to an orbital diagonal or crystal field basis. The advantage is twofold: first, the much smaller number of hybridization functions leads to fewer artificial correlations in the NCA solution, and second, we need to solve only one impurity problem per layer since the two Green's functions of the unit cell become identical by symmetry. We benchmarked the robustness of our simplified self-consistency approach against a full $\mathbf{k}$ summation over the Brillouin zone and an exactdiagonalization (ED) solver which, despite suffering from the discretization of the hybridization function that prevents the Hubbard bands from being smooth, are not influenced by any approximation and yield the exact $U / h$ ratio. The results of the comparison are reported in Fig. 1(a) [Fig. 1(b)], where we show the equilibrium DMFT spectral functions $A(\omega)=$ $-\operatorname{Im}\{\operatorname{Tr} \hat{G}(\omega)\} / \pi$ for LVO (YTO) in the bulk setup.

For LVO we choose $U=4.5 \mathrm{eV}$ and $J_{H}=0.64 \mathrm{eV}$, which are in accordance with previous studies [28,29] and result in a gap of the correct magnitude. For YTO we fixed the Hund's coupling to $0.64 \mathrm{eV}$ and searched for the $U$ parameter that best reproduces the experimental spectral gap of $0.8 \mathrm{eV}[14,15]$. This procedure yields $U=3.5 \mathrm{eV}$. However, with this value of the interaction parameter, the equilibrium calculation with the simplified self-consistency and NCA solver gives a metallic solution, forcing us to increase the interaction strength to $U=3.75 \mathrm{eV}$. This latter value yields the spectral functions of Fig. 1(b) (thick line). In the case of LVO, the approximate treatment for $U=4.5 \mathrm{eV}$ gives good agreement with the ED result, which indicates, as expected, that the approximate self-consistency and NCA are more reliable when electronic correlations are stronger. Both results agree remarkably well with regard to the widths and substructures of the upper and lower Hubbard bands (UHB and LHB, respectively). This demonstrates that our simplified treatment provides a qualitatively correct description of the high-energy feature of the systems. All the following calculations are performed at temperature $T=0.1 \mathrm{eV}$, which is small compared to the gap size and the characteristic energy scales of the system.

\section{Light-matter coupling}

If the multiorbital basis is not complete, as is usually the case if only a subset of orbitals is considered, the coupling between the electromagnetic field and matter is, in general, not gauge invariant [30]. A possible solution is to express the light-matter interaction in terms of the physical fields and to start from the hopping Hamiltonian in the continuous space and dipolar approximation: $\mathcal{H}_{0}+\mathbf{E}(t) \cdot \mathbf{D}$. Here, $\mathbf{D}=e \mathbf{r}$ is the dipolar matrix that we project onto the Wannier basis. The effect of the light on the system is then given by a time-, orbital-, and site-dependent hopping [31]

$$
\begin{aligned}
h_{\alpha \beta}^{a b}\left(\mathbf{R}_{i}, t\right)= & e^{i \frac{e}{\hbar} \mathbf{A}(t)\left(\mathbf{R}_{i}+\mathbf{r}_{b}-\mathbf{r}_{a}\right)} \\
& \times\left[h_{\alpha \beta}^{a b}\left(\mathbf{R}_{i}\right)+\mathbf{E}(t) \cdot \mathbf{D}_{\alpha \beta}^{a b}\left(\mathbf{R}_{i}\right)\right],
\end{aligned}
$$

with $\mathbf{A}(t)=-\int_{0}^{t} d s \mathbf{E}(s)$ being the vector potential, $\{\alpha, \beta\}$ being the orbital indexes, and $\mathbf{r}_{a, b}$ being the relaxed coordinates of the sites $\{a, b\}$ within the unit cell defined by $\mathbf{R}$. To mimic a solarlike excitation one could Fourier transform the solar spectrum, but we will scan different photon energies $\Omega$ in order to study the effect of impact ionization. We apply 10-fslong in-plane electric field pulses with Gaussian power spectra $f_{\mathrm{G}}$ centered on different $\Omega$ and amplitudes adjusted such that the number of photons is the same for all the pulses. We used $|\mathbf{E}(\Omega, \omega)|^{2} \sim f_{\mathrm{G}}(\omega-\Omega)$, with $\int d \omega|\mathbf{E}(\Omega, \omega)|^{2} / \omega=$ const. In the following analysis we consider the weak-field limit, in which we found the dipolar matrix to have a negligible effect.

\section{RESULTS}

\section{A. Impact ionization in bulk setups}

We start by investigating the carrier multiplication induced by impact ionization [32] in bulk LVO and YTO. Our result can be understood considering that, in the NCA approximation, the solution is given by an expansion around the atomic limit; therefore, the quantum numbers (density $N$, spin $S$, and angular momentum $L$ ) of the local Hamiltonian are conserved quantities [33]. The Hamiltonian is block shaped, where different sectors correspond to different fillings $N$ of the impurity. The degenerate eigenstates belonging to each sector, with the same spin and orbital angular momentum, can be grouped together and collectively denoted as $\psi_{n}(N)$, where $n$ labels the energy level of the degenerate subgroup. Our model of LVO contains two electrons in three orbitals; hence, the equilibrium ground state is dominated by $N=2$ states, which, according to Hund's rule, are in a high-spin configuration. Photoexcitation primarily creates charge carriers in the $N=1$ and $N=3$ sectors. In the following, we will denote these long-lived charge carriers as "singlons" (holelike excitation, $N=1$ ), and "triplons" (electronlike excitation, $N=3$ ). According to the same nomenclature, in YTO, which has a ground state in the $N=1$ sector, the charge carriers will be "holons" (holelike excitation, $N=0$ ) and "doublons" (electronlike excitation, $N=2$ ). Both compounds host three available subgroups of eigenstates for the particlelike excitations and one for the holelike one, explaining the shape of the spectral functions reported in Fig. 1.

A pulse with an energy comparable to the gap will create a singlon at the top of the LHB and a triplon in a high-spin configuration at the bottom of the UHB. A more energetic pulse can populate one of the two low-spin configurations of the $N=3$ sector. These states store a considerable amount of Hund energy, of the order of $5 J_{H}$, which can be released by flipping back to the high-spin state, and potentially produce additional singlon-triplon pairs. We call this mechanism, which is conceptually related to singlet fission in molecular systems [6], "Hund impact ionization" (HII). The second impact ionization channel involves charge carriers with high kinetic energy scattering from the upper to the lower edge of a Hubbard (sub)band, exciting additional singlon-triplon pairs. This mechanism will be referred to as "kinetic impact ionization" (KII) [32]. Both HII and KII are expected to play a role in the LVO structure since $5 J_{H}$ and the widths of the $\psi_{n}(N=3)$ subbands are comparable to or larger than the gap.

A sketch of how HII occurs in LVO is shown in Fig. 2(a): the pulse initially excites low-spin configurations, which quickly decay to the high-spin state. Already from this cartoon we deduce that a side effect of the presence of HII is the decrease of the density of doubly occupied orbitals 

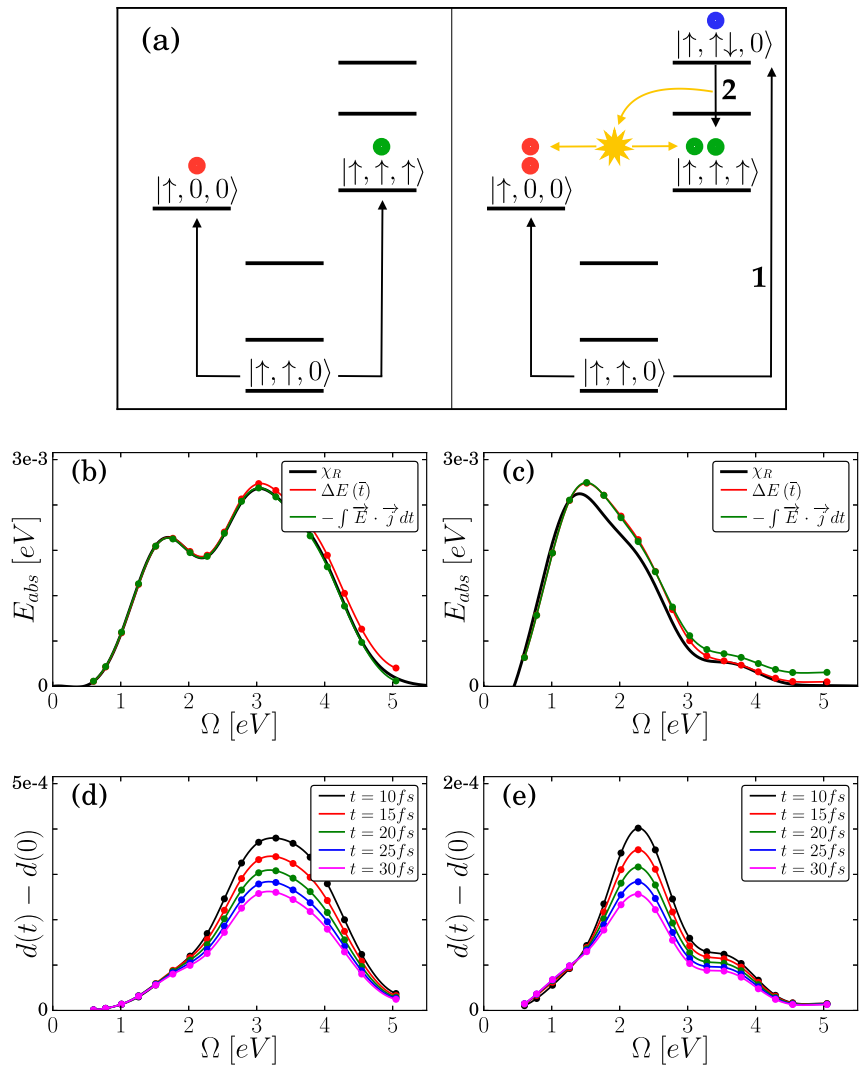

FIG. 2. (a) Illustration of the HII process in LVO. Left: lowenergy pulses produce only high-spin triplons. Right: high-energy pulses initially excite low-spin configurations (1), which quickly decay to the high-spin state (2). The energy released by the latter process can produce an additional singlon-triplon pair. Absorption spectra of (b) LVO and (c) YTO computed with different methods: weighted convolution between Hubbard bands (black), energy variation after the pulse (red), and integral of absorbed power (green). (d) and (e) Change in the double occupancy $d(t)$ relative to the initial value.

$d(t)=\sum_{\alpha}\left\langle\hat{n}_{\alpha \uparrow} \hat{n}_{\alpha \downarrow}\right\rangle(t)$ with time and a corresponding increase in the probability of high-spin configurations. This can occur only if the frequency of the exciting photon is enough to populate the high-energy states. In order to assess the relevance of this physics, we first compute the absorbed energy per site of LVO and YTO in their bulk setups with three different methods: (i) as the convolution between the LHB and the UHB, weighted by the photon probability distribution, $\chi_{R}(\Omega)=\int \frac{d \omega}{\omega} f_{\mathrm{G}}(\omega-\Omega) \int d \omega^{\prime} A_{\mathrm{LHB}}\left(\omega^{\prime}\right) A_{\mathrm{UHB}}\left(\omega-\omega^{\prime}\right)$, where $f_{\mathrm{G}}(\omega-\Omega)$ is the power spectra of the pulse; (ii) as the total energy increase $\Delta E_{\text {kin }}+\Delta E_{\text {pot }}$ right after the pulse at $t=10 \mathrm{fs}$; and (iii) as the integral of the injected power. The results are shown in Figs. 2(b) and 2(c). Both compounds start to significantly absorb energy as soon as $\Omega$ overcomes the gap, with a distribution that strongly depends on how the occupied part of the spectrum overlaps with the unoccupied part. The absorption in LVO is higher for high-energy photons due to the larger spectral weight associated with low-spin configurations, a consequence of the high multiplicity of the $\psi_{1}(N=3)$ and $\psi_{2}(N=3)$ eigenstates [33]. For YTO the spectral distribution is different, yielding a stronger absorption for smaller $\Omega$. The information on the energy range in which HII is active can be deduced from Figs. 2(d) and 2(e), where we plot the density of photoinduced double occupancies at different time slices. Even though we excite two different ground states, with filling $N=2$ for LVO and $N=1$ for YTO, the particlelike excitations in the charge sector contain three families of states in both cases. And in both cases the excited states with the lowest energy are high-spin configurations that do not contain any double occupancy $\left(\psi_{0}\right)$, while the families of states at higher energy $\left(\psi_{1}\right.$ and $\left.\psi_{2}\right)$ host lowspin configurations containing doubly occupied orbitals. Any process associated with HII must then be accompanied by a reduction of $d(t)$ happening on the same timescale as the carrier multiplication. Comparing Figs. 2(d) and 2(e) with Figs. 2(b) and 2(c), one sees that the reduction occurs in the energy range compatible with a population of the two highenergy families of states in both systems. This demonstrates the presence of a low- to high-spin decay in YTO but with a considerably smaller magnitude than in the case of LVO (note the different $y$-axis scales). In particular, the process involving the low-spin state with the highest energy, which is the one producing the largest carrier multiplication effect in HII, is almost absent. This behavior is a direct consequence of the position and width of the peaks within the UHB, proving that the gap amplitude is not the only relevant parameter that needs to be tailored to take advantage of impact ionization. A direct way to study the charge multiplication effect is to consider the time-dependent contribution $w_{n}(N, t)$ of each group of eigenstates to the trace of the density matrix, which can be measured directly within NCA. $w_{n}(N, t)$ contains the information on how, during the pulse and the following relaxation, weight is shifted between the different families of atomic eigenstates, resulting in the time dependence of local observables. In LVO an increasing $\Delta w_{0}(1, t)=w_{0}(1, t)-w_{0}(1,0)$ indicates singlon production, while an increasing $\Delta w_{n}(3, t)$ corresponds to triplon production in the family of states associated with $n$. We present this analysis only for LVO since for YTO the variations are much smaller and comparable to the numerical accuracy. In Fig. 3(a) we plot $\Delta w_{0}(1, t)$ and $\Delta w_{0,1,2}(3, t)$ for photon energies corresponding to excitations from the LHB to the three peaks of the UHB. Exciting triplons into a low-energy, high-spin configuration $\psi_{0}(3)$ leads to a long-lived state without any carrier multiplication within the accessible time window, as one can infer from the almost constant weights. On the other hand, as soon as one of the two low-spin configurations is populated, the system quickly (even during the pulse) relaxes to the $\psi_{0}(3)$ state. This relaxation leads to a triplon/singlon population that increases in time, consistent with HII. The time derivatives plotted in Fig. 3(b) (measured at $t=12 \mathrm{fs}$ ) show that HII is activated for pulse energies $\Omega$ larger than $2 \mathrm{eV}$, i.e., as soon as the low-spin states are populated. Since the transition is between two local states, HII does not necessarily result in a lowering of the kinetic energy $K$. Spin state transitions (and the field pulse itself) may produce carriers at the upper edge of the high-spin subband $\psi_{0}(3)$. This enables the transformation of kinetic energy into additional singlon-triplon pairs via KII. In Fig. 3(c) we show the kinetic energy variation $\Delta K(t)=K(t)-K(0)$ with respect to the equilibrium result. Note that a negative $\Delta K(t)$ corresponds to an increase of the kinetic energy (which 

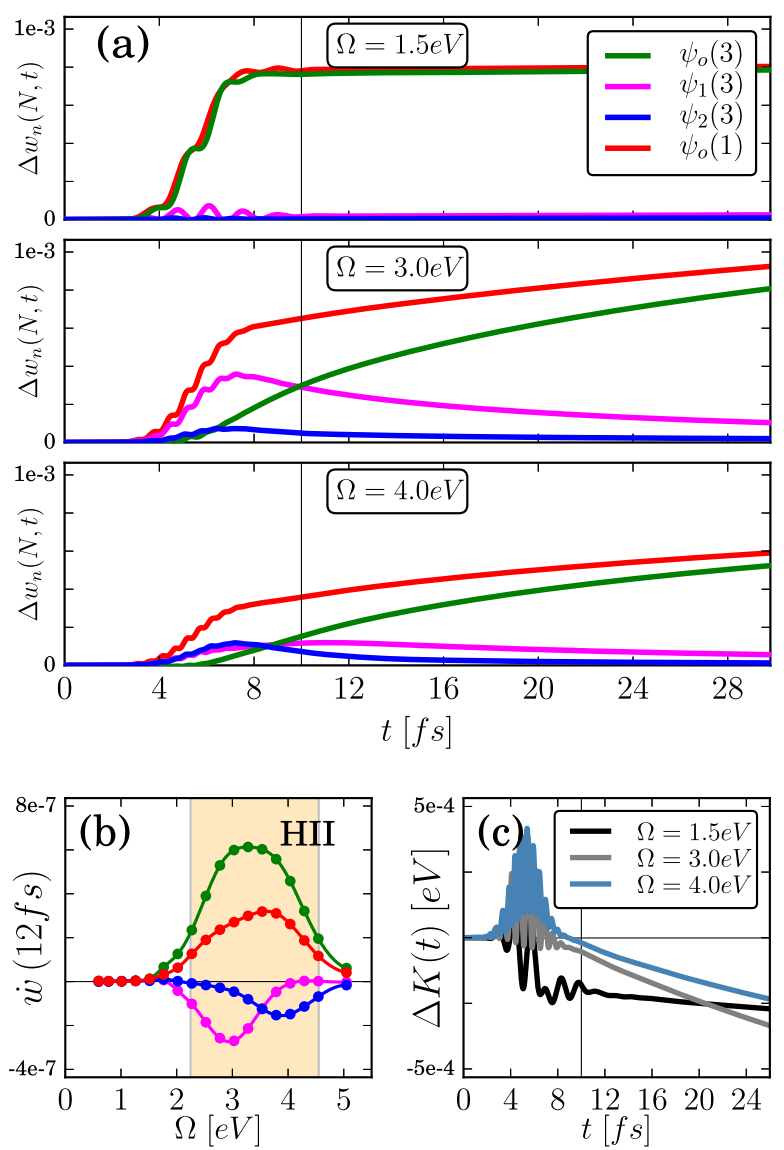

FIG. 3. (a) Time evolution of the singlon and triplon weights $\Delta w_{n}(N, t)$ for pulses of energy $\Omega$ promoting carriers to each of the three triplon subbands. The straight vertical line marks the end of the pulse. (b) Time derivative of $\Delta w_{n}(N, t)$ at $t=12 \mathrm{fs}$. Negative values correspond to decaying high-energy triplon states; positive values correspond to accumulating singlon and low-energy triplon states. HII is active in the shaded region. The color code is the same as in the legend in (a). (c) Change in kinetic energy $\Delta K(t)$ after the pulse. All these results refer to LVO.

is a negative quantity) that follows from the injection of additional carriers into the system. After the low-energy pulse ( $\Omega=1.5 \mathrm{eV}$ ), only KII is active, and we observe a rapid decrease of the kinetic energy during and immediately after the pulse, while the HII processes triggered by higher-energy pulses repopulate the high-kinetic energy states, which results in a slower decrease of $K(t)$.

These results show that the two processes, HII and KII, are intertwined, with HII boosting KII, and that the carrier multiplication by impact ionization occurs on the timescale of a few tenths of a femtosecond. On this short timescale, energy dissipation to phonons can be neglected.

\section{B. Carrier separation in heterostructured LVO and YTO}

To study how photoinduced carriers are separated by a potential gradient we construct multilayers composed of four LVO (YTO) layers and two STO layers, thereby modeling semi-infinite STO substrates on both sides of the film. We note that a larger number of STO layers does not significantly

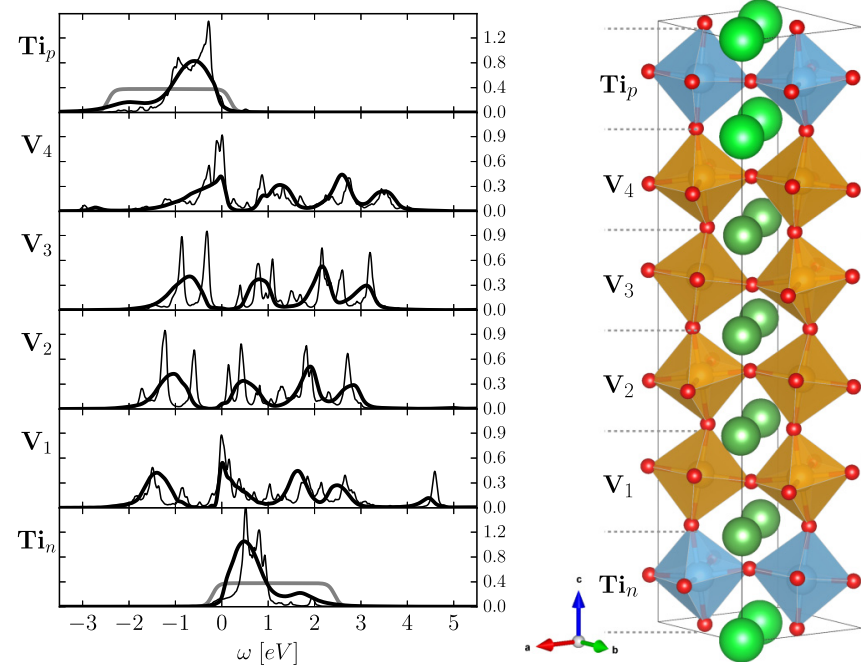

FIG. 4. Comparison between the spectral functions obtained by the full $\mathbf{k}$ summation and an ED solver (thin black lines) and those obtained with the approximate self-consistency and NCA solver (thick black lines) for the LVO/STO heterostructure. The gray lines indicate the DOS of the metallic leads. A unit cell of the LVO/STO heterostructure is shown on the right, where $\mathrm{La}(\mathrm{Sr})$ atoms are shown in dark (light) green, $\mathrm{V}$ is in orange, $\mathrm{Ti}$ is in blue, and $\mathrm{O}$ is in red. $\mathbf{T i}_{n}$ indicate the $n$-type interface $\left(\left[\mathrm{TiO}_{2}\right] /[\mathrm{LaO}]^{+}\right)$, and $\mathbf{T i}_{p}$ indicate the $p$ type $\left(\left[\mathrm{VO}_{2}\right]^{-} /[\mathrm{SrO}]\right)$. The latter has been shifted as described in the text.

alter the hoppings within LVO (YTO), which is the main information extracted from $a b$ initio DFT that is used in the subsequent nonequilibrium DMFT calculations. The internal field gradient, which results in metallic interfaces, has been computed with a separate DFT $+U$ calculation. This DFT setup, however, is not suitable for the study of charge separation for two reasons: (i) with empty Ti orbitals on both sides, the collection of holes at the top surface of the structure would be prevented. (ii) The $c$-axis periodicity in the supercell setup will prevent any charge extraction from the system, as carriers leaving the heterostructure on one side will reenter from the other. In order to overcome these limitations we empirically shifted down the local energy of the uppermost STO to allow for hole accumulation and replace the hopping between the two STO layers with a hopping into a noninteracting electron bath with flat DOS. The main role of this bath is to absorb carriers which flow out of the structure, therefore mimicking a semi-infinite metallic bulk. (A realistic setup resembling this system would likely involve a third component.) The equilibrium spectral function for our LVO/STO system is reported in Fig. 4. In the leads, represented by the three noninteracting Tilike $t_{2 g}$ bands and the noninteracting electron bath, the kinetic energy of the collected charge carriers will be dissipated since the chemical potential of the equilibrium structure is zero. To harvest energy, it is necessary to apply an external bias $V_{\text {bias }}$, which counteracts the internal field gradient, in order to collect the electron and holelike carriers at different chemical potentials. In the simulations, starting from an equilibrium state, we smoothly switch on the bias up to a constant value and wait for a few femtoseconds to allow the charge in the 


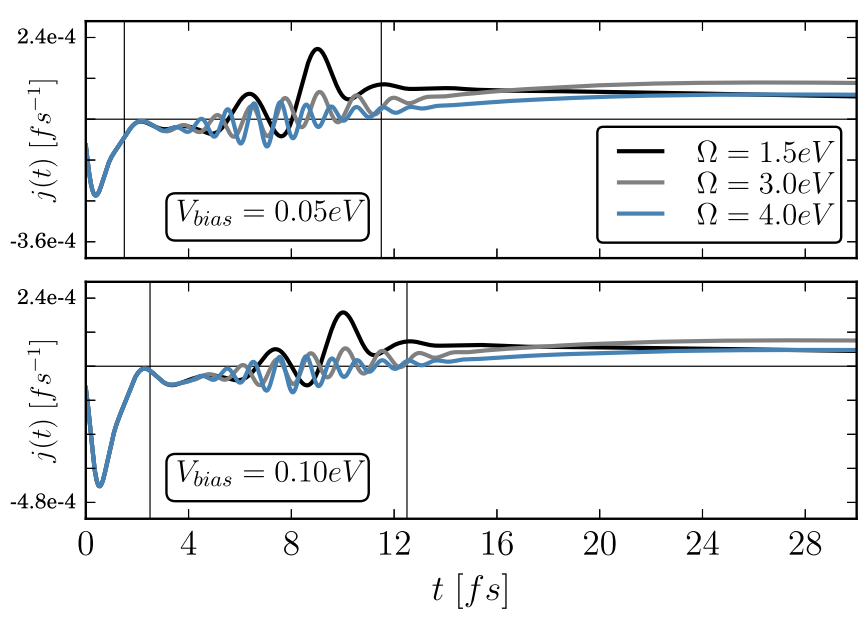

FIG. 5. Particle current density $j(t)$ flowing between the middle layers $\left(V_{2}, V_{3}\right)$ of the LVO/STO heterostructure of Fig. 4 with applied $V_{\text {bias }}=0.05 \mathrm{eV}$ and $V_{\text {bias }}=0.10 \mathrm{eV}$. The initial negative trend is due to the charge rearrangement induced by the bias (polarization current). The two vertical lines indicate the beginning and end of the pulse. The current decreases after the pulse for low-energy photons (black line), while, for more energetic photons (gray and light blue lines), the current continues to increase.

structure to rearrange before applying the pulse. Figure 5 shows, for a given bias and photon energy, the total current density $j(t)=-i\left[\Delta_{i}^{\perp} * G_{i}^{<}\right](t, t)$ which is flowing between the middle layers $\left(V_{2}\right.$ and $\left.V_{3}\right)$ for the LVO/STO system. The latter is measured via the DFT-derived hybridization function $\Delta_{i}^{\perp}$ defined in Eq. (2). Positive values of $j(t)$ indicate triplons moving from the top to the bottom of the heterostructure and singlons in the opposite direction. After an initial backward flow induced by the change of polarization occurring as a response to the increasing bias, the pulse produces charge carriers that will be eventually collected in the Ti leads. This current clearly reflects the carrier multiplication process described above: only high-energy photons $(\Omega>2 \mathrm{eV})$ result in a carrier production and an increasing interlayer current after the pulse (see, e.g., gray and light blue curves in Fig. 5). The effect of impact ionization manifests itself in an increase of the charge flowing out of the device as a function of pulse energy $\Omega$ on timescales that we can reach only by extrapolation. To better characterize the energy-harvesting process, we define the energy ratio

$$
\eta(t)=\frac{V_{\text {bias }}}{E_{\text {abs }}} \int_{0}^{t}\left[j^{\mathrm{Ti}^{\mathrm{top}}}\left(t^{\prime}\right)+j^{\mathrm{Ti} \mathrm{i}^{\mathrm{bottom}}}\left(t^{\prime}\right)\right] d t^{\prime},
$$

where $E_{\text {abs }}$ is the energy absorbed by the system right after the pulse and $j^{\mathrm{Ti}}(t)$ is the current between the Ti layer and the adjacent bath. $\eta(t)$ measures the fraction of the absorbed energy that is converted into extractable potential energy at time $t$ for the given $V_{\text {bias }}$. The voltage bias affects the energy-harvesting process in two ways: it increases the harvested energy per unit of charge and influences the charge separation process by counteracting the internal field [34]. Increasing $V_{\text {bias }}$ helps open transport channels by decreasing the DOS mismatch between neighboring layers and preventing high-energy carriers from being localized by the strong internal field [12]. Since at the longest time that we can reach the interlayer currents
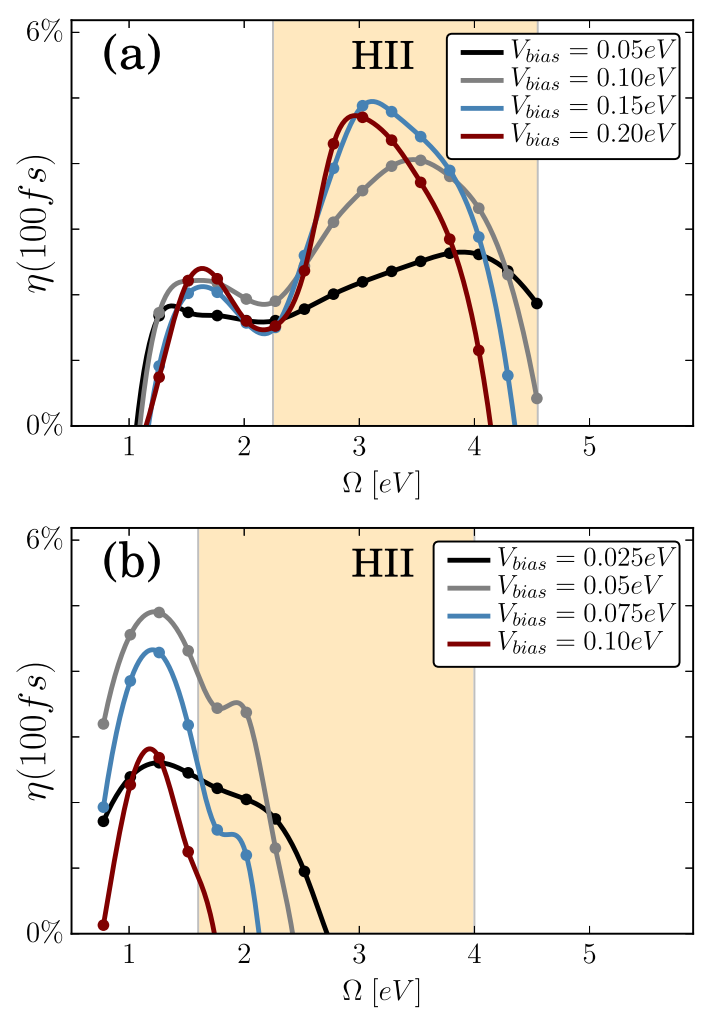

FIG. 6. (a) and (b) Extractable energy fraction at $t=100 \mathrm{fs}$ for the LVO- and YTO-based heterostructures, respectively. Pulse energies near $\Omega>2 \Delta_{g}$ eV result in HII (shaded region).

are still influenced by the (negative) polarization current, we extrapolate the harvested energy fraction to $100 \mathrm{fs}$ using the exponential fit $\eta_{\text {fit }}(t)=A\left[1-\exp \left(t_{0}-t\right) / \tau\right]$. In Figs. 6(a) and $6(\mathrm{~b})$ we report the result for the two heterostructures as a function of pulse energy $\Omega$. Both systems show a comparable peak at low energy corresponding to the first charge excitation, which is always overlapping with the UHBs of the neighboring layers. However, only LVO/STO exhibits a significant extractable energy fraction when $\Omega>2 \Delta_{g}$. This is a consequence of the fact that, as we showed in the bulk analysis, in YTO the carrier multiplication associated with impact ionization is negligible. Only in LVO/STO does $\eta$ at high $\Omega$ increase with increasing $V_{\text {bias }}$ : this behavior is not a consequence of a higher absorption since we normalize by $E_{\text {abs }}$, but rather of the increase in the carrier concentration induced by impact ionization and of the improved alignment of the adjacent UHBs. An increase of $V_{\text {bias }}$ beyond $0.15 \mathrm{eV}$ in LVO/STO is detrimental to the harvesting process as the peak in $\eta$ shrinks to a narrower energy window. This threshold value for the breakdown voltage of the device is smaller for YTO/STO as a consequence of the smaller gap. Eventually, the current leaving the system will vanish since the produced charge carriers are either extracted by the leads or lost by recombination. As we show in Fig. 5, the current starts to drop right after the pulse for a low-energy photon, while it continues to increase up to the maximum simulation time when carrier multiplication occurs. As a consequence, one may expect that the second peak in $\eta$ continues to increase much beyond $6 \%$, in contrast to the first one, which at $100 \mathrm{fs}$ already represents the total extracted charge. Figure 6(a) 


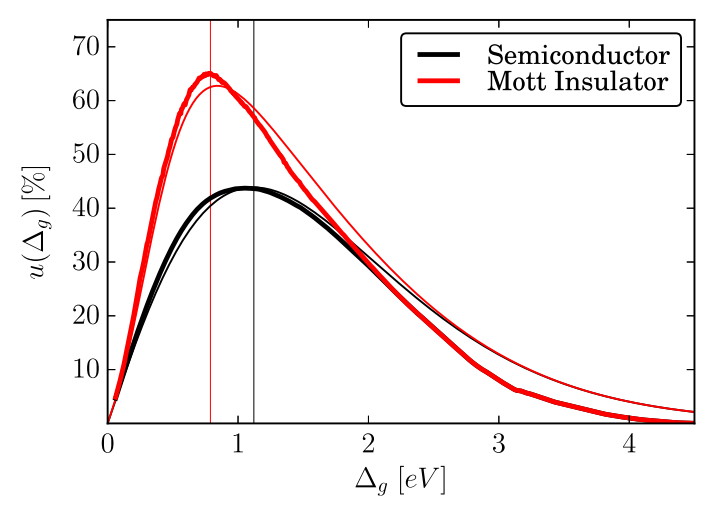

FIG. 7. Ultimate efficiency of a semiconductor solar cell (black) and Mott solar cell (red). The thin and thick lines show results for the black body and solar spectrum, respectively. The straight vertical lines indicate the location of the optimal gap amplitude for the solar spectrum.

hence demonstrates that impact ionization plays an important role in converting high-energy photons into additional harvestable charge carriers, thus contributing to a potentially high efficiency of Mott solar cells.

\section{Optimal gap size}

In the LVO-based compounds, HII becomes relevant for photon energies in the tail of the solar light spectrum [9]. Since impact ionization requires pulse energies larger than twice the gap, we thus conclude that the optimal gap size for Mott solar cells has to be smaller than $1.1 \mathrm{eV}$. The SQ estimate for the ultimate efficiency $u\left[\Delta_{g}\right]$ of semiconductor solar cells assumes that all charge excitations across a gap of size $\Delta_{g}$ contribute only an energy $\Delta_{g}$ regardless of the spectral distribution. In the presence of impact ionization, a simple generalization of the SQ argument yields

$u\left[\Delta_{g}\right]=\frac{\Delta_{g}\left[\sum_{i=1}^{M} i \int_{i \Delta_{g}}^{(i+1) \Delta_{g}} d \omega N(\omega)+\int_{(M+1) \Delta_{g}}^{\infty} d \omega N(\omega)\right]}{\int_{0}^{\infty} d \omega N(\omega) \omega}$.

Here, we use a rather crude estimate of the effect of impact ionization, in the same spirit of the original SQ calculation, considering that photons with energy $\Omega=2 \Delta_{g}$ generate twice the carriers of their low-energy counterparts and those with $\Omega=3 \Delta_{g}$ generate three times more. In the formula we assumed $M=3$ available excitations in the charge sector, which is consistent with our compounds. In Fig. 7 we compare the original and Mott versions of the ultimate efficiency as a function of the gap size for photon densities $N(\omega)$ corresponding to black body radiation at $6000 \mathrm{~K}$ (thin line) and sunlight (thick line). The optimal gap size for Mott solar cells is approximately $0.8 \mathrm{eV}$, which would be, indeed, compatible with YTO $[14,15]$. However, we showed that in YTO most of the spectral weight is concentrated in the high-spin sector, and for this reason HII plays a minor role. Still, because the main absorption peak is at lower energy, this compound may be of interest for solar cell applications.

\section{CONCLUSIONS}

We presented a semirealistic study of the device characteristics of LVO- and YTO-based Mott solar cells. While it would be instructive to include the (empty) $e_{g}$ states, which lie within an energy window accessible to the solar light spectrum, we do not expect these states to contribute substantially to impact ionization. Therefore, our model captures the relevant physical processes that motivate the interest in Mott insulating compounds for solar cell applications, namely, the carrier multiplication effects via HII and KII. In particular, we demonstrated the importance of HII for the harvesting of high-energy photons and the cooperative effect between HII and KII, which can result in a significant increase in the density of charge carriers on the 10 -fs timescale, which is extremely short compared to decay processes associated with phonon excitations. From this we conclude that impact ionization should, indeed, contribute to the efficient harvesting of solar energy in Mott systems [4], provided that photoinduced carriers are efficiently separated before recombination. Our results point to an important role of the substructures of the Hubbard bands which result from local atomic physics. In principle, a lower Hund's coupling would shift the Hubbard subbands associated with low-spin configurations to lower energies, which would be beneficial for light-harvesting purposes. However, $5 J_{\mathrm{H}}$ needs to be large compared to typical phonon energies to avoid competition from phonon relaxation.

\section{ACKNOWLEDGMENTS}

This work was supported by the Swiss National Science Foundation through NCCR MARVEL and the European Research Council through ERC Consolidator Grant No. 724103. The calculations were performed on the $\mathrm{Beo} 04$ cluster at the university of Fribourg and the Piz Daint cluster at the Swiss National Supercomputing Centre (CSCS), using a software library developed by M. Eckstein and H. Strand. F.P. thanks Adriano Amaricci for useful discussions on the ED calculations, which were performed with a code developed at SISSA [35].
[1] K. T. A. Kojima, Y. Shirai, and T. Miyasaka, J. Am. Chem. Soc. 131, 6050 (2009).

[2] J.-H. Im, C.-R. Lee, J.-W. Lee, S.-W. Park, and N.-G. Park, Nanoscale 3, 4088 (2011).

[3] M. Assadi, S. Bakhoda, R. Saidur, and H. Hanaei, Renew. Sustain. Energy Rev. 81, 2812 (2018).

[4] E. Manousakis, Phys. Rev. B 82, 125109 (2010).
[5] L. Zhang, Y. Zhou, L. Guo, W. Zhao, A. Barnes, H.-T. Zhang, C. Eaton, Y. Zheng, M. Brahlek, H. F. Haneef, N. J. Podraza, M. W. Chan, V. Gopalan, K. M. Rabe, and R. Engel-Herbert, Nat. Mater. 15, 204 (2016).

[6] A. J. Nozik, M. C. Beard, J. M. Luther, M. Law, R. J. Ellingson, and J. C. Johnson, Chem. Rev. 110, 6873 (2010).

[7] M. B. Smith and J. Michl, Chem. Rev. 110, 6891 (2010). 
[8] W. Shockley and H. J. Queisser, J. Appl. Phys. 32, 510 (1961).

[9] E. Assmann, P. Blaha, R. Laskowski, K. Held, S. Okamoto, and G. Sangiovanni, Phys. Rev. Lett. 110, 078701 (2013).

[10] L. Wang, Y. Li, A. Bera, C. Ma, F. Jin, K. Yuan, W. Yin, A. David, W. Chen, W. Wu, W. Prellier, S. Wei, and T. Wu, Phys. Rev. Appl. 3, 064015 (2015).

[11] M. Jellite, J.-L. Rehspringer, M. Fazio, D. Muller, G. Schmerber, G. Ferblantier, S. Colis, A. Dinia, M. Sugiyama, A. Slaoui, D. Cavalcoli, and T. Fix, Solar Energy 162, 1 (2018).

[12] M. Eckstein and P. Werner, Phys. Rev. Lett. 113, 076405 (2014).

[13] H. Aoki, N. Tsuji, M. Eckstein, M. Kollar, T. Oka, and P. Werner, Rev. Mod. Phys. 86, 779 (2014).

[14] Y. Okimoto, T. Katsufuji, Y. Okada, T. Arima, and Y. Tokura, Phys. Rev. B 51, 9581 (1995).

[15] I. Loa, X. Wang, K. Syassen, H. Roth, T. Lorenz, M. Hanfland, and Y.-L. Mathis, J. Phys.: Condens. Matter 19, 406223 (2007).

[16] P. Giannozzi, S. Baroni, N. Bonini, M. Calandra, R. Car, C. Cavazzoni, D. Ceresoli, G. L. Chiarotti, M. Cococcioni, I. Dabo, A. D. Corso, S. de Gironcoli, S. Fabris, G. Fratesi, R. Gebauer, U. Gerstmann, C. Gougoussis, A. Kokalj, M. Lazzeri, L. Martin-Samos, N. Marzari, F. Mauri, R. Mazzarello, S. Paolini, A. Pasquarello, L. Paulatto, C. Sbraccia, S. Scandalo, G. Sclauzero, A. P. Seitsonen, A. Smogunov, P. Umari, and R. Wentzcovitch, J. Phys.: Condens. Matter 21, 395502 (2009).

[17] J. P. Perdew, K. Burke, and M. Ernzerhof, Phys. Rev. Lett. 77, 3865 (1996).

[18] We checked carefully that the discrepancy of the in-plane mesh is not relevant for the consecutive analysis.
[19] A. A. Mostofi, J. R. Yates, Y.-S. Lee, I. Souza, D. Vanderbilt, and N. Marzari, Comput. Phys. Commun. 178, 685 (2008).

[20] N. Marzari, A. A. Mostofi, J. R. Yates, I. Souza, and D. Vanderbilt, Rev. Mod. Phys. 84, 1419 (2012).

[21] M. Eckstein and P. Werner, Phys. Rev. B 88, 075135 (2013).

[22] A. Georges, G. Kotliar, W. Krauth, and M. J. Rozenberg, Rev. Mod. Phys. 68, 13 (1996).

[23] H. Keiter and J. C. Kimball, J. Appl. Phys. 42, 1460 (1971).

[24] M. Eckstein and P. Werner, Phys. Rev. B 82, 115115 (2010).

[25] M. Eckstein, T. Oka, and P. Werner, Phys. Rev. Lett. 105, 146404 (2010).

[26] M. Ligges, I. Avigo, D. Golež, H. U. R. Strand, Y. Beyazit, K. Hanff, F. Diekmann, L. Stojchevska, M. Kalläne, P. Zhou, K. Rossnagel, M. Eckstein, P. Werner, and U. Bovensiepen, Phys. Rev. Lett. 120, 166401 (2018).

[27] P. Werner, H. U. R. Strand, S. Hoshino, and M. Eckstein, Phys. Rev. B 95, 195405 (2017).

[28] M. De Raychaudhury, E. Pavarini, and O. K. Andersen, Phys. Rev. Lett. 99, 126402 (2007).

[29] G. Sclauzero and C. Ederer, Phys. Rev. B 92, 235112 (2015).

[30] B. A. Foreman, Phys. Rev. B 66, 165212 (2002).

[31] D. Golež, L. Boehnke, M. Eckstein, and P. Werner, Phys. Rev. B 100, 041111(R) (2019).

[32] P. Werner, K. Held, and M. Eckstein, Phys. Rev. B 90, 235102 (2014).

[33] L. de' Medici, J. Mravlje, and A. Georges, Phys. Rev. Lett. 107, 256401 (2011).

[34] It also affects the polarization current at short times, but that is not relevant for the characterization of a real device.

[35] https://github.com/aamaricci/dmft-ed. 